\title{
USO DE DIPIRONA EN PACIENTES HOSPITALIZADOS
}

\author{
Rocío Moreno de Santacruz *, Carmen Yaneth Buitrago**, Margarita Pérez Pérez ***
}

\section{Resumen}

La dipirona es un analgésico y antipirético muy usado en Colombia; es considerado en otros países como medicamento perjudicial. El objetivo de este trabajo es determinar su uso como analgésico para el manejo del dolor en una entidad de tercer nivel. Estudio descriptivo de corte transversal, con una población de 240 adultos que recibieron tratamiento con dipirona; las fuentes de datos son la historia clínica, el consumo reportado por farmacia en el año 2011 y el cálculo mensual de las dosis diarias definidas. El análisis estadístico de la información se realizó en Stata 10. Se encontró que este fármaco es el analgésico de primera línea en la institución para el manejo del dolor en el postoperatorio, con dosis de prescripción superior a la establecida por la OMS. Se analiza la posible relación con reacciones adversas, que se presentaron en $52 \%$ de los pacientes, como fueron hipotensión $(98 \%)$ y erupción cutánea $(2 \%)$. Se concluye que hay necesidad de ajustar la dosis a 3 g/día para observar la efectividad clínica del medicamento y el impacto económico en la institución.

Palabras clave: dipirona, efectos de drogas, dosis, farmacovigilancia.

Abreviaturas: DDD, dosis diaria definida.

\section{USE OF DIPYIRONE IN HOSPITALIZED PATIENTS}

\section{Abstract}

Dipyrone is an analgesic and antipyretic broadly used in Colombia; in other countries it is considered a harmful drug. This work aimed to determine its use in pain management in a tertiary health care facility. A descriptive cross sectional study was conducted in a population of 240 adults who received dipyrone therapy; data was obtained from clinical records, pharmacy reports of its use in year 2011 and a monthly estimate of defined daily doses. Stata 10 was used for data statistical analysis. The study revealed that dipyrone is used as a first line analgesic for postoperative pain management in the institution, using higher prescription doses than those established by the WHO. The potential relationship with adverse reactions was analyzed, and was found in $52 \%$ : hypotension (98\%) and skin rash (2\%). It was concluded that the dose needs to be adjusted to $3 \mathrm{~g} /$ day in order to observe the analgesic efficacy of dipyrone and its financial impact on the institution.

Key words: dipyrone, drug effects, dose, pharmacovigilance (Drug Safety)

Fecha recibido: febrero 25 de 2013 - Fecha aceptado: mayo 24 de 2013

* Enfermera especialista en gerencia de la salud. Profesora Titular, Fundación Universitaria de Ciencias de la Salud. Bogotá DC, Colombia.
*** Enfermera especialista en cuidado del adulto en situaciones críticas. Profesora Titular, Fundación Universitaria de Ciencias de la Salud. Bogotá DC, Colombia.

** Enfermera especialista en gerencia de la salud. Profesora Asistente, Fundación Universitaria de Ciencias de la Salud. Bogotá DC, Colombia. 


\section{Introducción}

La dipirona es un derivado pirazolónico no narcótico, perteneciente al grupo de los analgésicos antiinflamatorios no esteroideos, que posee propiedades analgésicas y antipiréticas con débil efecto antiinflamatorio. ${ }^{1} \mathrm{Su}$ mecanismo de acción se basa en la inhibición de la síntesis de prostaglandinas a nivel periférico. Se utiliza en pacientes con fiebre que no responden a otros antipiréticos como el acetaminofén, en dolores agudos e intensos postraumáticos, postoperatorios o de origen tumoral como en cáncer. ${ }^{2}$

La potencialidad del medicamento de producir efectos adversos ${ }^{3}$ varía desde muy raros $(0.01 \%)$ como es el caso de la agranulocitosis, hasta muy frecuentes (mayor de 10\%) como son urticaria, exantema medicamentosa, diaforesis, edema, disnea, broncoespasmo e hipotensión, por lo cual se hace necesaria la vigilancia de la duración del tratamiento que debe ser inferior a siete días. ${ }^{1}$ Por esta capacidad de producir efectos adversos con consecuencias, en la década de los setenta este medicamento salió del mercado en varios países americanos. Peter Schönhöfer señala su prohibición en Alemania en 1981 y anota que la dipirona sódica es un medicamento de alto riesgo para la salud por el debilitamiento del sistema inmunológico, o agranulocitosis, que puede llegar a producir la muerte. ${ }^{4}$ Hamerschlak, Maluf, Biasi y col. en 2005 en el estudio multinacional de casos y controles en países americanos concluyen que la agranulocitosis inducida es imprevisible e independiente de la dosis. ${ }^{5}$ Otros estudios argumentan que la condición puede ser genética, racial o que requiere de otros factores de riesgo para su aparición. ${ }^{6,7}$ Por otra parte, Macias EM, Ruiz A, Moreno y col. en 2007, en su investigación indican que el uso de la dipirona está muy extendido en España por su favorable farmacocinética y su efecto analgésico, similares a lo obtenida con dosis baja de opiáceos, con ventajas adicionales como son no causar complicaciones gastrointestinales graves y tener una ligera acción relajante sobre los músculos. ${ }^{8}$ Esta diversidad de posturas frente a la conveniencia o no de utilizarla hace imprescindible su farmacovigilancia para promover la seguridad clínica de los pacientes. No obstante Walter Vasen y cols. señalan que esta es una herramienta poco utilizada por los médicos, los químicos farmacéuticos y las enfermeras en las instituciones ${ }^{9}$, lo que puede incidir en el uso inadecuado debido a dosis mayores o menores de las recomendadas, tiempos inadecuados de tratamiento o falta de análisis de costo/efectividad. Por otra parte, para evaluar la utilización por período de los medicamentos en las instituciones, la OMS desde 1981 sugiere el método de las dosis diarias definidas (DDD), el cual es claro y de fácil aplicación. Se basa en fuentes confiables de información sobre el volumen de uso de los medicamentos por período, la DDD por expertos para el medicamento y el número de camas ocupadas en el mismo período. La DDD corresponde a la dosis promedio diaria de un medicamento, aplicado a un adulto en una determinada forma farmacéutica. ${ }^{10}$ Para el cálculo del total de las DDD se suma el total de gramos o unidades internacionales utilizados en un período de tiempo y se divide por la DDD de los expertos para el medicamento en estudio, luego se divide por el número de camas ocupadas en el mismo período y se multiplica por cien. Es un indicador que sirve para comparar el uso de fármacos en distintos períodos, servicios u hospitales, pero no tiene una interpretación clínica. ${ }^{11}$

En Colombia, el Instituto de Vigilancia de Medicamentos y Alimentos INVIMA, ha reglamentado que la dipirona debe ser utilizada bajo fórmula médica como analgésico de segunda opción, en casos de dolor o fiebre moderados o intensos que no han cedido a alternativas farmacológicas previas (analgésicos no narcóticos); restringe la forma parenteral de manera ambulatoria y su uso en instituciones prestadoras de servicios de salud debe ser prescrito por un especialista. Si la vía parenteral se prolonga por más de siete días debe realizarse control con hemograma para asegurar un monitoreo continuo con el fin de detectar la aparición de agranulocitosis. ${ }^{12}$ La $O M S$ estableció como DDD para la dipirona $3 \mathrm{~g} /$ día $^{12}$, y en la literatura se encuentra como dosis máxima 6 a 8 g/día. ${ }^{13}$

Dado lo anterior, es indiscutible que la enfermera como integrante del equipo de salud que tiene mayor contacto con el paciente, responsable de evaluar e informar al médico la respuesta al tratamiento, incluyendo la terapia farmacológica, tenga juicio clínico 
para observar los efectos deseados o adversos de los medicamentos. A pesar de ser una de las actividades que ocupa la mayor parte del tiempo y exige pleno conocimiento y seguridad, a menudo surgen dudas sobre la preparación y administración que no siempre son consultadas, lo que puede relacionarse con el inadecuado manejo de las prescripciones en las

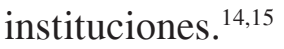

Como el hospital donde se realizó el estudio no dispone de una guía de analgesia para este medicamento, el objetivo del trabajo es determinar el uso de la dipirona como analgésico en el manejo del dolor en el área de hospitalización de pacientes adultos, para fundamentar la necesidad de establecer la guía de analgesia en la que se incluyan aspectos como la valoración de la escala del dolor, el seguimiento a las reacciones adversas, tiempo de tratamiento, uso racional y efectividad clínica, utilizando la DDD fijada por la OMS.

\section{Método}

Estudio descriptivo de corte transversal. Se seleccionaron las historias clínicas de 240 pacientes que cumplieron con los criterios de inclusión: mayores de 18 años, hospitalizados, que recibieron tratamiento farmacológico con dipirona, con cualquier diagnóstico de ingreso; 117 en el período de agosto 2011 y 123 en noviembre de 2011 para aumentar el número de sujetos observados. Los criterios de exclusión fueron mujeres gestantes y pacientes con patología hematológica. Se revisaron las órdenes médicas, registro de medicamentos y notas de enfermería durante el tiempo que los pacientes recibieron dipirona y permanecieron hospitalizados. Se indagó con el jefe del servicio de farmacia de la institución la presentación disponible del fármaco. Para el cálculo de la DDD se tomó como referencia la determinada por la OMS que es igual a $3 \mathrm{~g} /$ día en el adulto, sin considerar los ajustes individuales que pueden ser necesarios de acuerdo con la condición clínica. El total de gramos utilizados por mes se obtuvo en el programa (software) del sistema Hipócrates del servicio de farmacia para la hospitalización de adultos; luego se indagó en el servicio de estadística los días/camas ocupadas mes a mes y se aplicó la siguiente fórmula: total de DDD en los servicios de hospitalización de adultos por período x 100/ número de días cama ocupadas en los servicios de hospitalización de adultos del mismo período.

La base de datos se construyó en Excel 2007. El análisis estadístico de la información se realizó en Stata 10. Para el análisis se sumaron los dos grupos; las variables cualitativas de edad, sexo y especialidad tratante se reportan en frecuencias absolutas y porcentajes, y las variables cuantitativas como fueron gramos de dipirona administrados por día, tiempo de prescripción y la clasificación en mayor, igual o menor a la dosis diaria definida por la OMS, se analizan con medidas de tendencia central. Los resultados se presentan mediante tablas de salida y figuras. Es una investigación sin riesgo aceptada por el comité de ética institucional y se cumplió el principio de confidencialidad de la información obtenida de la historia clínica de los pacientes.

\section{Resultados}

En 2011 ingresaron al área de hospitalización médicoquirúrgica 12.936 pacientes y fueron despachadas por farmacia 77.892 ampollas de dipirona magnésica de dos gramos. La población de los períodos observados estuvo constituida por 133 hombres $(55,4 \%)$ con edad media de 51 años y 107 mujeres $(44,6 \%)$ cuya media fue 53 años.

La dipirona se utilizó como primera opción para el manejo del dolor posoperatorio por las especialidades quirúrgicas (Tabla 1). El 96,6 \% recibió más de tres gramos al día (Tabla 2). La mediana del tiempo de tratamiento con este analgésico fue tres días. De acuerdo con los registros clínicos de enfermería el 47,9\% de los pacientes (115) presentaron hipotensión (98\%) y erupción cutánea (2\%) durante el período en que se administró la dipirona. La institución solo dispone de la presentación magnésica de dos gramos. De acuerdo con el resultado del cálculo de consumo de dipirona se observa que en la mayor parte del año (8 meses), la probabilidad por cada 100 camas ocupadas/día de que un paciente sea formulado con este medicamento es de 100\% (Figura 1). 


\begin{tabular}{|c|c|c|}
\hline Especialidad & $\mathrm{N}^{\circ}$ de pacientes & Porcentaje \\
\hline Cirugía general & 138 & 57,5 \\
\hline Ortopedia & 65 & 27,08 \\
\hline Neurocirugía & 15 & 6,25 \\
\hline Medicina interna & 8 & 3,33 \\
\hline Otros & 14 & 5,84 \\
\hline Total & 240 & 100 \\
\hline
\end{tabular}

Agosto y noviembre de $201 \mathrm{I}$.

Tabla 2. Perfil de prescripción de la dipirona en el área de hospitalización medicoquirúrgica

Dosis y frecuencia de administración
$\mathrm{N}^{\circ}$ de pacientes
Porcentaje

2 g cada 8 horas

$2 \mathrm{~g}$ cada 6 horas 158

65,8

I g cada 8 horas

74

30,8

Agosto a noviembre de $201 \mathrm{I}$

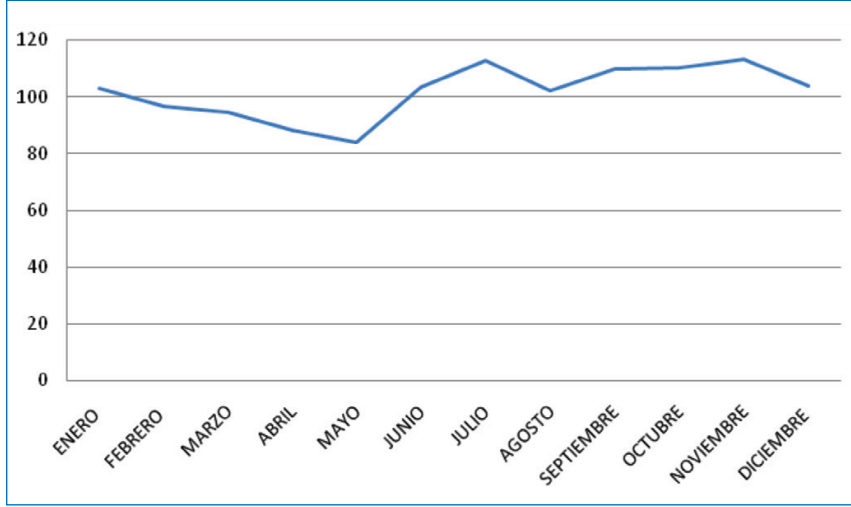

Figura I. Consumo de dipirona de acuerdo con la dosis diaria definida en el área medicoquirúrgica de una entidad de tercer nivel de Bogotá DC, año 20I I.

Fuente: informe de farmacia sobre el consumo mensual de dipirona (20II).

\section{Discusión}

Los resultados del estudio confirman lo encontrado por Edwards, Meseguer, Faura, Moore y McQuay ${ }^{16}$ sobre los estados clínicos en los cuales se utiliza la dipirona: pacientes con dolor postoperatorio agudo de moderado a intenso, en el tipo cólico, el producido por cáncer y en casos de migraña. El informe de Montoya, Vaca y Parra ${ }^{17}$ sobre reacciones adversas con dipirona revela que se prescribió como analgésico en $96 \%$ y en $4 \%$ como antipirético.

Aunque no se observaron casos de angioedema o anafilaxia como los descritos en el trabajo de Schnyder ${ }^{18}$, si ocurrieron tres de urticaria. La hipotensión de los pacientes registrada durante el tiempo en que se les administró la dipirona puede estar relacionada con la velocidad de infusión descrita en el consenso de expertos mexicanos, los cuales explican este descenso abrupto de la tensión arterial porque la activación de la vía óxido nítrico-GMP cíclico-canales de potasio produce vasodilatación. ${ }^{1}$ En el estudio no se realizó seguimiento riguroso a las interacciones farmacológicas con otros medicamentos ni a la técnica de su administración. Se identificó que la farmacovigilancia no es una fortaleza en la institución, debido a que ninguno de los signos presentados por los pacientes que podrían estar relacionados con reacciones adversas, fue reportado por los médicos tratantes ni enfermeras que se encontraban a cargo al comité de seguridad clínica o de farmacovigilancia. La única presentación disponible de dos gramos de dipirona magnésica en la institución ocasiona desperdicio cuando se formula un gramo. En el hospital se utilizan en promedio 101,81 dosis diarias definidas por cada 100 camas en un día, lo que confirma el sobreuso observado en los registros de las historias clínicas, donde se identificó que 158 pacientes recibieron tres gramos diarios más y 74 cinco gramos diarios más que la dosis recomendada ( $3 \mathrm{~g} /$ día) por la OMS.

En conclusión, la frecuente prescripción de dipirona en los servicios quirúrgicos con dosis diarias superiores a la definida por la OMS, la falta de seguimiento sistemático y continuo por el médico y enfermeras ante la utilización de la dipirona y el riesgo de posibles efectos adversos para el paciente, demuestran la necesidad de conformar un equipo multidisciplinario para establecer la guía de analgesia, en especial para los pacientes que se encuentran en postoperatorio con prescripción de dipirona para el manejo del dolor. Se recomienda incluir la presentación de un gramo en el vademécum hospitalario, así como disponer de dipirona sódica y hacer seguimiento al costoefectividad clínica, con el ajuste a tres gramos diarios del medicamento. 


\section{Referencias}

1. Arcila H, Barragán S, Borbolla J, Canto A, Castañeda G,et al . Consenso de un grupo de expertos mexicanos: Eficacia y seguridad del metamizol (Dipirona). Gac. Méd.Méx.2004; 140(1):99-101.

2. Hamerschlak N, Cavalcanti AB.. Neutropenia, agranulocytosis and dipyrone. Sao Paulo Med J. 2005 Sep 1;123(5):247-9.

3. Maj S, Lis Y. The incidence of metamizole sodium-induced agranulocytosis in Poland. J Int Med Res. 2002 Sep-Oct; 30(5):488-95.

4. Lemus I, Pinardi G, Schönhöfer P. Advierten sobre riesgos de la dipirona [Monografía en Internet]. Santiago, Chile: PadresOk; 2011 [citado 19 Jun 2013]. Disponible en: http://www.padresok.com/2011/01/advierten-sobre-riesgos-dela-dipirona/

5. Kaufman DW, Kelly JP, Levy M, Shapiro S. The drug etiology of agranulocytosis and aplastic anemia. New York: Oxford University Press; 1991.

6. Risks of agranulocytosis and aplastic anemia. A first report of their relation to drug use with special reference to analgesics. The International Agranulocytosis and Aplastic Anemia Study. JAMA 256(13):1749-57.

7. Siles Gutiérrez M, Ávila Muñoz L, Gómez Juanes V. Sistema de codificación de principios activos y dosis diarias definidas del INSALUD [Monografía en Internet]. Madrid, España: INSALUD; 2002 [citado 19 Jun 2013]. Disponible en: http://www.ingesa.msssi.gob.es/fr/estadEstudios/documPublica/pdf/codificacion.pdf

8. Macías E, Ruiz A, Moreno E, Laffond E, Dávila I, Lorente F. Usefulness of intradermal test and patch test in the diagnosis of nonimmediate reactions to metamizol. Allergy. 2007 Dec;62(12):1462-4.

9. Vasen W, Fiorentino RM. Farmacovigilancia una herramienta poco utilizada Medicina (Buenos Aires). 2006; 66(3): 257-62.
10. Guía internacional de indicadores de precios de medicamentos [Monografía en Internet]. Cambridge, Massachusetts: MSH; 2011 [citado 19 Jun 2013]. Disponible en: http://erc.msh.org/dmpguide/pdf/DrugPriceGuide_2011_sp.pdf

11. Tipos de estudio de utilización de medicamentos [Monografía en Internet]. Santiago, Chile: Universidad de Chile; 2011 [citado 19 Jun 2013]. Disponible en: http://mazinger.sisib.uchile.cl/repositorio/lb/ciencias_quimicas_y_farmaceuticas/arancibiaa01/articulo28/b.html

12. Farmacovigilancia [Monografía en Internet]. Bogotá: INVIMA; 2012 [citado 19 Jun 2013]. Disponible en: http://web.invima.gov.co/portal/faces/index. jsp?id=4575

13. OMS. Agranulocitosis asociada con la Dipirona. Ginebra: Organización Mundial de la Salud; 2008.

14. Blasco Segura P, Mariño E.L, Aznar Saliente M.T, Pol Yanguas E, Almiñana MA, Castels Molina M, Velasco Álvarez M.L. Desarrollo de un método observacional prospectivo de estudio de Errores de Medicación para su aplicación en hospitales. Farm Hosp. 2001; 25(5): 253-73.

15. Otero MJ, Domínguez-Gil A. Acontecimientos adversos por medicamentos: una patología emergente. Farm Hosp. 2000; 24:258-66.

16. Edwards JE, Messenger F, Faura C, Moore RA, McQuay HJ. Single dose dipyrone for acute renal colic pain. Cochrane Database Syst Rev. 2002;(4): CD003867.

17. Montoya GA, Vaca C, Parra MF. Detección de efectos secundarios asociados a la administración de tramadol y dipirona en un hospital de alta complejidad. Biomédica 2009 Jul-Sep; 29(3):369-81.

18. Schneider CH, Kasper MF, de Weck AL, Rolli H, Angst BD. Diagnosis of antibody mediated drug allergy. Pyrazolone and pyrazolidinedione crossreactivity relationship. Allergy .1987 Nov;42(8):597-603. 